\title{
Can Taste Rating of Groundwater Samples for the Presence of Iron Be A Novel Approach to Groundwater Iron
}

\section{Assessment?}

Sabuktagin Rahman ${ }^{1 *}$, Malay Kanti Mridha ${ }^{2,3}$, Patricia Lee ${ }^{4}$, and Faruk Ahmed ${ }^{1,5}$

${ }^{1}$ Public Health, School of Medicine, Gold Coast Campus, Griffith University QLD 4222, Australia

${ }^{2}$ Rang-Din Nutrition Study, Rajabasor, Parbotipur, Dinajpur, Bangladesh

${ }^{3}$ James P. Grant School of Public Health, BRAC University, Dhaka, Bangladesh

${ }^{4}$ Public Health, School of Medicine, Gold Coast Campus, Griffith University QLD 4215, Australia

${ }^{5}$ Menzies Health Institute Queensland, Australia

*Corresponding author: Sabuktagin Rahman

Public Health, School of Medicine, Gold Coast Campus, Griffith University QLD 4222, Australia, email: sabuktagin.rahman@griffithuni.edu.au, rahman.sabuktagin@gmail.com

\section{Abstract}

Groundwater has been shown to contribute markedly to the daily iron intake of the rural Bangladeshi population and is currently characterized as an under-assessed possible source of dietary iron. Estimation of the levels of iron in groundwater in relation to dietary/nutritional assessments has been called for. However, the ability to do this may be limited due to unavailable equipment or technical, logistical and financial issues in remote areas of low- to middle-income countries. Groundwater with higher levels of iron has distinctive organoleptic properties such as a characteristic metallic/bitterly taste and reddish color. Anecdotal experience suggests that there is an association between a stronger metallic taste of water and its iron content. Therefore, we conducted a cross-sectional pilot study assessing the relationship of taste perception for iron in groundwater and its actual concentration of iron. Thirteen tube-wells were selected systematically in a rural village of northern Bangladesh. A brief structured interview was conducted with a systematic sampling of people living nearby to collect information on the tube-wells, perception of the taste of water from the wells, and their overall perception of the level of iron in the water. Two observers from the research team tasted a water sample from each well for iron and compared their observations. Iron concentration of the tube-well water was determined quantitatively by a test kit (Hach kit model 18B). The concentration of iron was significantly higher in water taste-rated by both the villagers and observers as "strong" for iron 
than in water taste-rated as having "some" iron. There were significant correlations between the taste-ratings of the two observers and between the observers and villagers. Bland-Altman plotting suggests that external observers are likely to provide valid and reproducible taste-ratings for the presence of iron in the water. A larger study is required to validate tasting as a cheap, simple and novel way to assess iron concentration in groundwater.

Key words: Groundwater Iron Assessment, Taste-rating, Bangladesh

\section{Background and rationale}

Groundwater iron has long been regarded as an agent causing operational difficulties for humans, e.g., clogging household water supplies, home appliances, and staining clothes and body parts, such as teeth and nails. High levels of iron in groundwater result in unpleasant esthetic experiences with water, e.g., bad taste, odor, and color. However, recent studies in Bangladesh have revealed that iron in groundwater is a good source of absorbable iron in humans, leading to good iron and hemoglobin status in the population (Merrill 2011; Rahman 2016). Based on these findings, policymakers have endorsed the importance of considering groundwater iron in anemia/micronutrient deficiency control programs (Institute of Public Health Nutrition and United Nation Children's Fund 2016). However, assessing iron in groundwater requires a rigorous technical procedure to follow (Merrill, personal communication) and has logistical and cost implications; more importantly the equipment is not readily available in most low-income countries. Groundwater with iron has a distinctive "metallic"/"bitter" taste. Anecdotal experience of local residents suggests that there is an association between a strong metallic taste of water and the iron content in water. Further, they report staining of clothes, teeth, nails when using groundwater. They thus often use pond or river water (i.e. surface water) for bathing and washing clothes. When local residents perceive that a well contains only a small amount of iron, such complaints are uncommon. Hence, their perception of iron content in water is complemented by the presence or absence of some adverse consequences.

The present pilot study was conducted to assess the relationship between taste-rating of groundwater samples for iron and its actual iron concentration. It may provide the inspiration for conducting a larger study to validate whether the simple act of taste-rating of water samples for iron is adequate for the assessment of the iron level in groundwater.

\section{Methods}

Thirteen participants with nearby tube-wells were selected systematically from Madai Khamar village in Lohanipara union (a cluster of villages) of Badarganj sub-district in northern Bangladesh. A village street was chosen at random from the main road of the village, leading to the bank of the river of the area. The first tube-well selected was at the bank of the river. After that, in the retrograde direction from the river bank, every third tube-well was selected until 13 were selected. The purpose of the study was described, and verbal consent of the participants (household head or his wife) was taken before the collection of data. Information was requested on the tube-well the household was using for drinking water, such as the depth of the well, his/her perception of the degree of iron present in the well water, his/her taste perception of iron 
in water and water drinking behaviors. The depth estimation was aided by asking the respondent the number of pipes used to sink the well, each of which has a standard length.

Perception of levels of iron in groundwater: The measurement tool was adopted from an existing 4-point scale that had been found to be able to predict iron status in groundwater accurately (Merrill 2010). In Merrill's study, respondents were asked by the field enumerator a simple question as, "How much iron do you think is in the water that you pump from this drinking tube well?” The response options in the hedonic scale were: none $=0$, a little amount $=1$, a medium $=2$ and a lot $=3$. Respondents were asked to base this on their overall perception of iron in water based on the organoleptic qualities and their personal experiences (taste, smell, the physical appearance of water, iron discoloration of utensils, clothes, teeth, etc.) (Merrill 2011).

We also used a hedonic scale but modified it to: no taste of iron=0, some taste of iron=1 and strong taste of iron=2. We chose a more limited number of options in the scale, because differentiation of too many grades of taste perception may be difficult and can lead to a higher level of subjective error. Our taste-rating tool was based only on taste perception.

Two observers from the research team tasted the water sample of each well and noted their observations. To avoid bias, this observation was completed before interviewing the household members.

Estimation of iron in groundwater samples: Before collecting the water sample, the selected tube-well was pumped for 5 minutes to purge any residual minerals that might have built up in the pipes and to have access to the deeper aquifer. A freshwater sample was collected directly into a $1 \mathrm{~L}$ plastic bucket rinsed with the respective tube-well water. Total iron concentration in this water was determined to the nearest $0.1 \mathrm{mg} / \mathrm{L}$ by the Hach kit model $18 \mathrm{~B}$ test kit using the FerroVer method (Merrill 2009) which includes 1,10-Phenanthroline as a reagent. This test kit method has been validated for iron in groundwater (Merrill 2009).

Statistical analysis: To determine the association between the depth of the tube-well and concentration of iron in its water, a curvilinear fit model was used. Since the perceived tasterating for iron levels in water is an ordinal (3-point) variable and iron concentration in water is a continuous variable, to assess the correlation of the observer's and the villager's taste-ratings of iron in water with its actual iron concentration, Spearman's rank correlation coefficient (Spearman's rho) were estimated (https://onlinecourses.science.psu.edu/stat509/node/157). To study the correlation of the ranks of the taste perception of iron in the tube-well water between the villagers vis-à-vis the observers and between the observers, Kendall's $\tau$ b coefficients were estimated, as there were many tied values over the corresponding ranks (http://www.statisticssolutions.com/kendalls-tau-and-spearmans-rank-correlation-coefficient/). Non-parametric tests were used to determine the statistical difference in the mean estimates of iron in the water. Data analysis was done with the statistical software, STATA 14 (STATA Inc. College Station, Texas, USA) and SPSS 18. 
Ethics approval: The study was conducted in preparation for a trial assessing the efficacy of a new formulation of micronutrient powder (MNP with a low dose of iron) in children of areas with high levels of iron in groundwater. The trial received approval from the Research Ethical Committee of Faculty of Biological Science, Dhaka University, Bangladesh and Griffith University Human Research Ethics Committee, Australia.

\section{Results}

Data were obtained for both observers for 13 tube wells, but from villagers for only 11 of them. Figure 1 illustrates the non-significant association between the depth of the tube-wells and iron concentration in its water (standardized $\beta=-2.07, \mathrm{p}=0.2$ ).

Figure 1: Relation ${ }^{1}$ of the depth of tube-well and concentration of iron (irnconc) in the groundwater sample

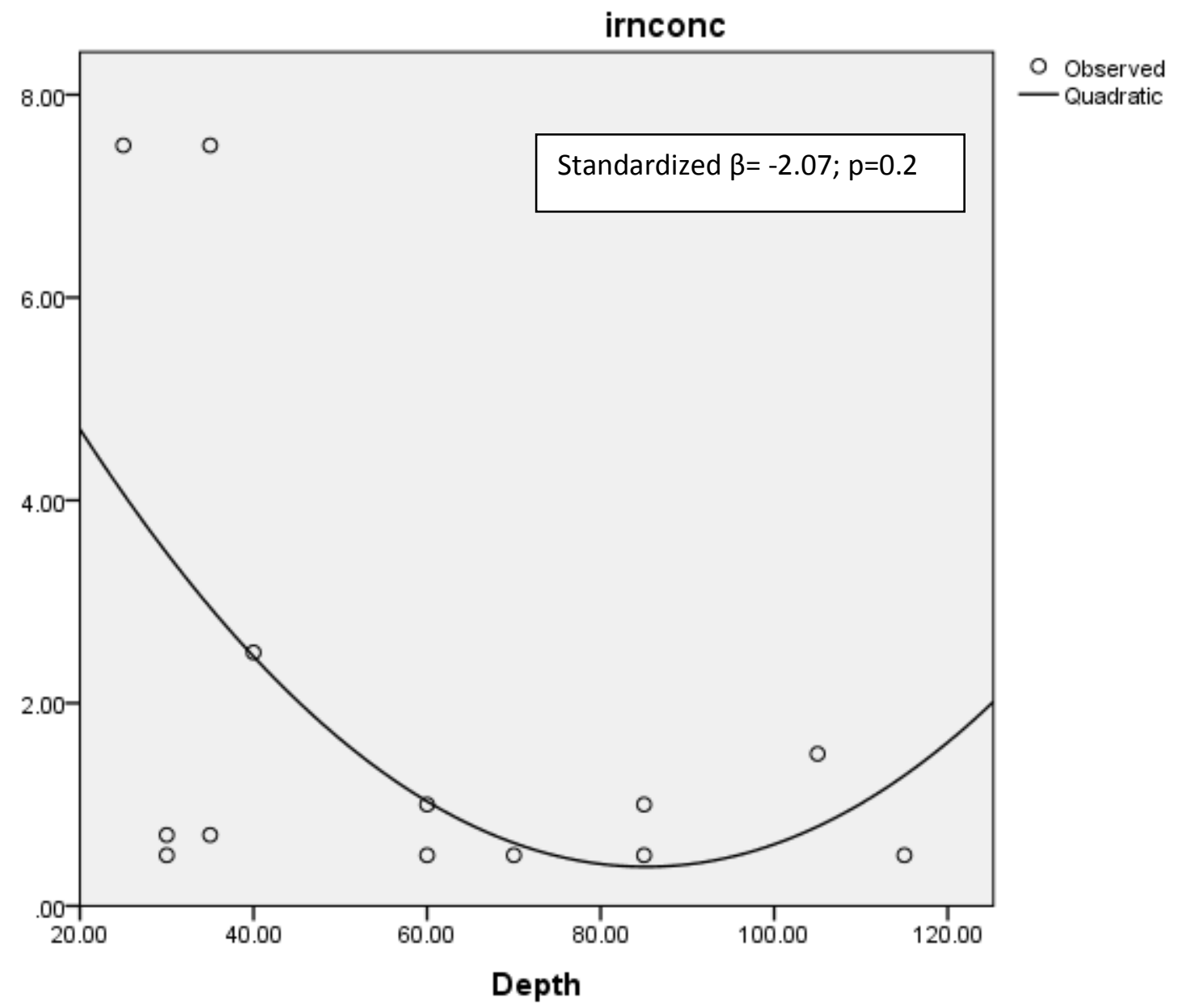

${ }^{1}$ Quadratic Curve-Fit 
Table 1 shows the taste-rating of the degree of iron in tube-well water and how it related to measured iron concentrations in the sample water. Among the villagers who tasted "some" level of iron in the water of their wells, the mean (SD) and median concentrations of iron in the water samples were $0.71(0.39) \mathrm{mg} / \mathrm{l}$ and $0.5 \mathrm{mg} / \mathrm{l}$ respectively. For the villagers who rated that their wells as having a "strong" taste of iron, the concentrations of iron in the water samples were 4.62 (3.37) $\mathrm{mg} / \mathrm{l}$ and $5.0 \mathrm{mg} / \mathrm{l}$ as mean (SD) and median respectively. As can be seen in Table 1, the values for the two observers showed similar trends.

Table 1: Taste rating for iron in groundwater and distribution of measured levels of iron

\begin{tabular}{|c|c|c|c|c|c|}
\hline \multirow{2}{*}{$\begin{array}{l}\text { Taste rating of } \mathrm{Fe}^{3} \\
\text { status in wells }\end{array}$} & \multicolumn{4}{|c|}{ Fe in tube well water } & \multirow[t]{2}{*}{ Test for Difference of Means } \\
\hline & $\mathrm{n}$ & Range & Mean(SD) & Median & \\
\hline Villagers & 11 & & & & \\
\hline "Some taste of Fe" & 7 & $0.5-1.5$ & $0.71(0.39)$ & 0.5 & \multirow{2}{*}{$\mathrm{P}=0.027^{1}$} \\
\hline "Strong taste of Fe" & 4 & $1.0-7.5$ & $4.62(3.37)$ & 5.0 & \\
\hline Observer 1 & 13 & & & & \\
\hline "Some taste of Fe" & 9 & $0.5-1.5$ & $0.71(0.34)$ & 0.5 & \multirow{2}{*}{$\mathrm{P}=0.006^{2}$} \\
\hline "Strong taste of Fe" & 4 & $1.0-7.5$ & $4.6(3.3)$ & 5.0 & \\
\hline Observer 2 & 13 & & & & \\
\hline "Some taste of Fe" & 10 & $0.5-2.5$ & $0.89(0.65)$ & 0.6 & \multirow{2}{*}{$\mathrm{P}=0.028^{2}$} \\
\hline "Strong taste of Fe" & 3 & $1.0-7.5$ & $5.33(3.75)$ & 7.5 & \\
\hline
\end{tabular}

${ }^{1}$ Independent Samples Kruskal Wallis Test

${ }^{2}$ Independent Samples Mann-Whitney U Test

${ }^{3} \mathrm{Fe}=$ iron

For the villagers as well as for the two observers, the mean of iron in "some" vs "strong" taste of iron was significantly different.

Table 2 depicts the correlation coefficents of taste-rating for the level of iron in tube-well water and measured iron concentration in the water samples. All had high and statistically significant correlations.

Table 2: Correlation of iron taste rating in tube-well water and measured concentration of iron

\begin{tabular}{ll}
\hline Variable & Iron concentration in tube-well water \\
\hline Villagers & Coefficient $^{1}$ \\
Observer 1 & $0.599^{*}$ \\
Observer 2 & $0.759^{* * *}$ \\
\hline
\end{tabular}

${ }^{*} \mathrm{p}=0.031,{ }^{* *} \mathrm{p}=0.021,{ }^{* * *} \mathrm{p}=0.003$

${ }^{1}$ Spearman rank correlation coefficient

Table 3 depicts the correlation matrix showing the association of taste-rating for iron in tubewell water among the villagers and the observers. These also were high and significant. 
Table 3: Correlation ${ }^{1}$ matrix showing association of taste-rating for iron in tube-well water among the villagers and observers

\begin{tabular}{llll}
\hline Variable & Villagers & Observer 1 & Observer 2 \\
\hline Villagers & 1 & $0.849^{* * *}$ & $0.697^{*}$ \\
Observer 1 & $0.849^{* * *}$ & 1 & $0.822^{* *}$ \\
Observer 2 & $0.697^{*}$ & $0.822^{* *}$ & 1 \\
\hline
\end{tabular}

${ }^{*} \mathrm{p}=0.012,{ }^{* *} \mathrm{p}=0.004,{ }^{* * *} \mathrm{p}=0.002$ (2-tailed)

${ }^{1}$ Kendall's $\tau$ b

Figure 2 shows Bland-Altman plots using the average of two ratings on the x-axis and the difference between those on the y-axis. The Bland-Altman comparison between the villagers and observer 1 (Grid A) shows the limits of agreement (reference range for difference) as -0.905 to 0.597 . The analysis shows the mean difference, -0.154 (95\% CI -0.381 to 0.073 ) and ranges from 0.5 to 2.0. Pitman's test of difference in variance is $r=0.576 ; \mathrm{p}=0.039$.

Figure 2: Bland-Altman Plots showing the limits of agreement between different assessors on the taste-rating of groundwater sample for the presence of iron

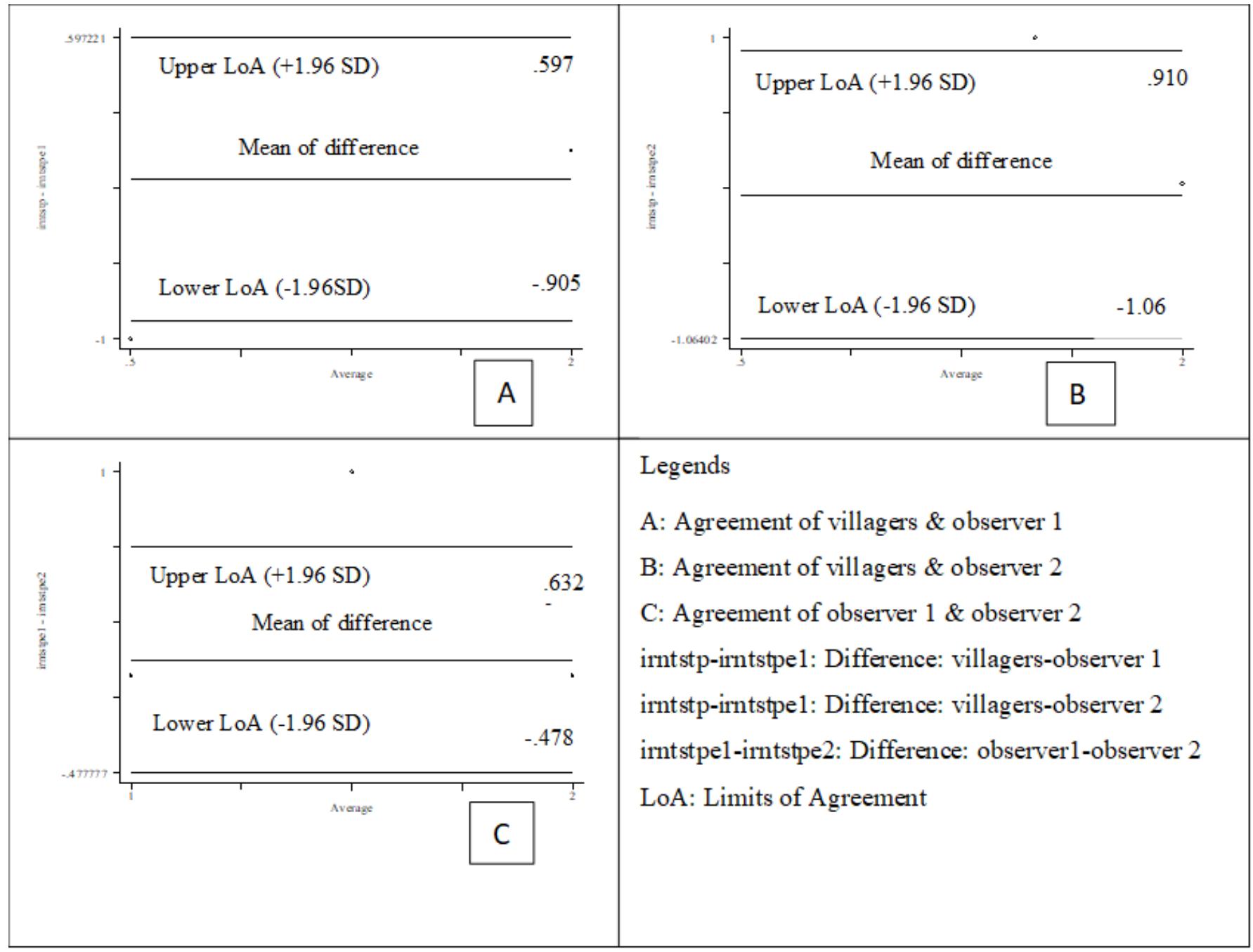


The Bland-Altman comparison between the villagers and observer 2 (Grid B) shows the limits of agreement ranged from -1.064 to 0.910 . The analysis further shows the mean difference of 0.077 (95\% CI -0.375 to 0.221 ) and the range from 0.5 to 2.0. Pitman's test of difference in variance reports the $r=0.547 ; p=0.053$. Bland-Altman comparison between observer 1 and 2 (Grid C) shows the limits of agreement ranges from -0.478 to 0.632 . Analysis reveals the mean difference of 0.077 (95\% CI -0.091 to 0.245) with a range from 1.0 to 2.0. Pitman's test of difference in variance reports $\mathrm{r}=0.158 ; \mathrm{p}=0.606$.

A 4-point module of the villager's overall perception of the degree of iron present in tube-well water samples and iron concentration showed a significant correlation; Spearman rho=0.76, $\mathrm{p}=0.003$ (data not shown).

\section{Discussion}

The present pilot study from a rural community of Bangladesh reports the relationship of tasteperception of the degree of iron present in the groundwater sample and the actual concentration of iron. It further examined the level of agreement between various raters about their perceptions of iron in the water, to determine whether in this setting taste-ratings by individuals has the potential to develop into a reliable tool for assessing levels of iron in groundwater. The study also compared the perception of the villagers of the level of iron in groundwater samples with the actual concentration of iron in the waters, using a modified version of a tool which had been used elsewhere in Bangladesh (Merrill 2009). Our finding of a positive relationship between the villager's perceived iron level and the actual amount of iron in the groundwater is in agreement with the other studies conducted in Bangladesh (Merrill 2009; Wendt 2016).

The analysis showed a non-significant negative association between the depth of the tube-wells and concentration of iron in its water, consistent with Bangladesh groundwater characteristics, where typically shallow wells have a higher concentration of minerals, such as iron and arsenic (DPHE/BGS 2001). The reason for the non-significant association could be due to the small sample size.

The wells that were rated to have tasted "strong" for iron had a significantly higher concentration of iron than the wells rated to have "some" taste of iron. This finding is in agreement with our assumption before embarking on the study that water with a stronger taste of iron might have a higher concentration of iron.

The Bland-Altman plot analysis is a simple way to evaluate a bias between the mean differences and to estimate an agreement interval, within which $95 \%$ of the differences of the second method, compared to the first one, fall (Giavarina 2015). As per the results of the Bland Altman analysis, a non-significant Pitman's test of difference in variance signifies that the measurements between the observer 1 and 2 are likely to be in acceptable agreement and reproducible. On the other hand, the significant Pitman's variances are indicative that the measurements between the villagers vs. observer 1 and the villagers vs. observer 2 are unlikely to be an acceptable agreement. The difference in ratings between the villagers vs. observers is likely due to habituation of the villagers in using iron-rich water, leading to under-estimation of the concentration of iron in their well water. Acceptable agreement between the observer 1 and 
observer 2 and apparently higher correlations of their taste-ratings for iron and actual iron concentrations in the water indicates that the external taste assessors could be more appropriate for assessment.

A limitation of the study is that it is based on a very small number of samples. As such, some instability in the findings cannot be ruled out. The results of the study yielded a relatively large SD around the mean estimates of iron concentration. A study with a larger sample size would likely have smaller variances. Nonetheless, observation of the expected direction of the associations and its consistency underlie the potential that the findings might be replicable in a larger study. The mean iron estimates sorted by different taste categories would also likely be more reliable, and thus has the potential to establish a reference iron concentration for groundwater organized by "some" and "strong" levels of taste categories for iron. The reference values can be used in relevant future studies, e.g., dietary assessments, iron/anemia program development and evaluations.

In conclusion, taste-rating of groundwater sample for the degree of presence of iron bears a positive association with the concentration of iron in the sample. External assessors are likely to provide valid and reproducible taste-rating for the presence of iron in the water. A larger study is required to validate the findings which might help to develop the taste-rating of the water sample as a cheap, simple and novel tool for a semi-quantitative assessment of iron in groundwater in the poor resource settings.

Acknowledgement: The study acknowledges the support of the Rang-Din Nutrition Study (RDNS) field office.

\section{Conflicts of Interest: None}

Authorship: S.R. and M.K.M. conceived the study. S.R. designed the study, S.R. and M.K.M. supervised data collection, S.R. analyzed data and wrote the manuscript, F.A., P.L. and M.K.M. provided critical review to finalize. S.R. has full access to all the data in the study and had final responsibility for the decision to submit for publication. All authors read and approved the final manuscript.

\section{References}

British Geological Survey \& Department for Public Health Engineering, Government of the People's Republic of Bangladesh (2001). Arsenic Contamination of Groundwater in Bangladesh. BGS Technical Report WC/00/19 [DG Kinniburgh and PL Smedley, editors]. Keyworth: BGS.

Giarvanina D (2015). Understanding Bland Altman analysis. Biochem Med (Zagreb) 25(2): 1411 doi: 10.11613/BM.2015.01551,

Merrill RD, Shamim AA, Ali H, Jahan N, Labrique AB, Schulze K, Christian P, West KP Jr (2011). Iron Status of Women Is Associated with the Iron Concentration of Potable Groundwater in Rural Bangladesh. Journal of Nutrition 141(5):944-949. doi: 10.3945/jn.111.138628. 
Merrill RD, Labrique AB, Shamim AA, Schulze K, Christian P, West, KP Jr (2010). Elevated and variable groundwater iron in rural northwestern Bangladesh. J Water Health; 8:818-825.

Merrill RD, Shamim AA, Labrique AB, Ali H, Schulze K, Rashid M, West KP Jr (2009).

Validation of two portable instruments to measure iron concentration in groundwater in rural Bangladesh. J Health Popul Nutr;27:414-8

Institute of Public Health Nutrition and United Nation Children's Fund (UNICEF) (2016). National Anemia Consultation Final Report, 24-25 July, Dhaka, Bangladesh: Institute of Public Health Nutrition and United Nation Children's Fund (UNICEF)

Rahman S, Ahmed T, Rahman AS, Alam N, Ahmed AMS, Ireen S, Chowdhury IA, Rahman SMM (2016). Determinants of iron status and Hb in the Bangladesh population: the role of groundwater iron. Public Health Nutrition Access 19:1862-1874

Wendt A, Waid J, Gabrysch S (2016). Perceived iron content in drinking water associated with anemia among women with iron-poor diets in rural Bangladesh, The FASEB Journal vol. 30 no. 1 Supplement 892.1 